\title{
Variaciones del color. Primeros estudios físico- químicos de las pinturas rupestres de La María, provincia de Santa Cruz
}

\author{
Ariel D. Frank, Lucas Gheco, Emilia Halac, Noemí Mastrangelo, Matías Landino, \\ Rafael S. Paunero y Fernando Marte
}

Recibido 20 de agosto 2019. Aceptado 25 de noviembre 2019

\begin{abstract}
"[Las cuevas de La María] merecen un relevamiento cuidadoso. Debe armarse una comisión para que realice este trabajo" (Alberto Rex González. Cuaderno de campo, 8 de marzo de 1975).
\end{abstract}

\begin{abstract}
RESUMEN
Presentamos los primeros resultados de los análisis físico-químicos realizados sobre pinturas rupestres de la localidad arqueológica La María, provincia de Santa Cruz. Implementamos un estudio microestratigráfico, el cual constituye una vía novedosa para el estudio de los pigmentos en el arte rupestre de la Patagonia meridional. Dicho abordaje incluye el análisis por microscopía electrónica de barrido con análisis elemental por espectroscopia dispersiva de rayos X y microespectroscopia Raman. Así, caracterizamos en términos elementales y moleculares los principales conjuntos tonales registrados en la localidad (rojos, blancos, amarillos y negros). Los resultados indican que la composición de las pinturas es heterogénea aun al interior de cada conjunto cromático. Se destaca el uso como pigmento de distintos óxidos de hierro para las pinturas rojas y amarillas, el empleo de óxido de manganeso y carbón para el negro y de sulfato de calcio y probablemente caolinita para el blanco. Asimismo, en algunos casos se habría empleado el yeso como carga. Por último, se describen los alcances, límites y desafíos futuros de esta investigación.
\end{abstract}

Palabras clave: Arte rupestre; Microestratigrafías; Composición; Patagonia meridional.

\begin{abstract}
COLOR VARIATION: INITIAL PHYSICO-CHEMICAL ANALYSES OF THE ROCK ART OF LA MARÍA, SANTA CRUZ PROVINCE. In this paper, the initial results of the physico-chemical analyses of the rock art of the La María archaeological locality, Santa Cruz province, are presented. A micro-stratigraphic approach, a novel methodology for the study of pigments in Southern Patagonia, was implimented. Samples were analyzed using scanning electron microscopy with energy-dispersive X-ray spectroscopy and micro-Raman spectroscopy. This methodology enabled the main colors found in the locality (red, white, yellow and black) to be characterized in elemental and molecular terms. Results show that the composition of the paintings

Ariel D. Frank. División Arqueología, Facultad de Ciencias Naturales y Museo, Universidad Nacional de La Plata (FCNyM, UNLP) y Consejo Nacional de Investigaciones Científicas y Técnicas (CONICET). Edificio Anexo Museo, laboratorio 107. 122 y 60 s/n, La Plata (1900), Buenos Aires, Argentina. E-mail: frank.ariel@gmail.com

Lucas Gheco. TAREA-Instituto de Investigaciones sobre el Patrimonio Cultural (IIPC), Universidad Nacional de San Martín (UNSAM) y Centro de Investigaciones y Transferencia de Catamarca (CITCA), CONICET-UNCA. Quinquela Martín 1784, Ciudad de Buenos Aires (1296), Argentina. E- mail: gheco@hotmail.com

Emilia Halac. Gerencia de Investigación y Aplicaciones. Comisión Nacional de Energía Atómica. Av. General Paz I499. Buenos Aires, Argentina. E-mail: halac@cnea.gov.ar

Noemí Mastrangelo. TAREA-IIPC, UNSAM. Martín 1784, Ciudad de Buenos Aires ( 1296), Argentina. E- mail: nmastrangelo@gmail.com Matías Landino. TAREA-IIPC, UNSAM. Martín 1784, Ciudad de Buenos Aires (1296), Argentina. E- mail: matiaslandino@gmail.com Rafael S. Paunero. División Arqueología, FCNyM, UNLP. 122 y 60 s/n, La Plata (1900), Buenos Aires, Argentina. E-mail: rpaunero@fcnym.unlp.edu.ar

Fernando Marte. TAREA-IIPC, UNSAM. Martín I784, Ciudad de Buenos Aires (1296), Argentina. E- mail: fmarte.iipc@gmail.com
\end{abstract}

Intersecciones en Antropología 21(1), enero-junio: 57-69. 2020. ISSN-e 1850-373X

https://doi.org/10.37176/iea.21.1.2020.530

Facultad de Ciencias Sociales - UNICEN - Argentina 
is heterogeneous, even within each tone. These data highlight the use of different iron oxides as pigments for red and yellow paintings. Manganese oxide and charcoal were used for black and calcium sulfate -- and probably kaolinite -- for white. In addition, gypsum was used as a filler in some cases. Finally, the scope, limits and future challenges of this research are discussed.

Keywords: Rock art; Micro-stratigraphies; Composition; Southern Patagonia.

\section{INTRODUCCIÓN}

La Patagonia meridional constituye una región importante en lo que refiere a manifestaciones rupestres. Estas son por lo general pinturas, realizadas en abrigos rocosos por las sociedades cazadoras-recolectoras que habitaron el área. Dichas manifestaciones han sido estudiadas sistemáticamente desde la década de 1950, en cuestiones vinculadas a los diversos estilos plasmados, su cronología, las superposiciones, las técnicas desarrolladas y su localización espacial (Gradin et al., 1979; Aschero, 1996; Paunero et al., 2005; Re, 2011, entre otros). Un aspecto que ha sido menos abordado refiere a las materias primas con las que fueron confeccionadas las pinturas (ver infra).

En este trabajo presentamos los primeros resultados de los análisis físico-químicos implementados en la localidad arqueológica La María, provincia de Santa Cruz (Figura 1). Realizamos estudios microestratigráficos de un conjunto de muestras de pinturas rupestres así como de rocas soporte, que fueron analizados por microscopía electrónica de barrido con análisis elemental por espectroscopia dispersiva de rayos X (SEMEDS) y microespectroscopia Raman (ER). Así, describimos los primeros datos que permiten caracterizar, en términos elementales y moleculares, los principales conjuntos tonales registrados en la localidad (amarillos, rojos, blancos y negros), discutimos los resultados y presentamos algunas posibles líneas de trabajo futuras. Buscamos generar información de base que nos permita, con el tiempo, no solo comprender con qué recursos se realizaron las pinturas, sino también que constituya una vía de entrada para abordar las tecnologías pictóricas y otros aspectos ligados a la dinámica de las sociedades que habitaron el área.
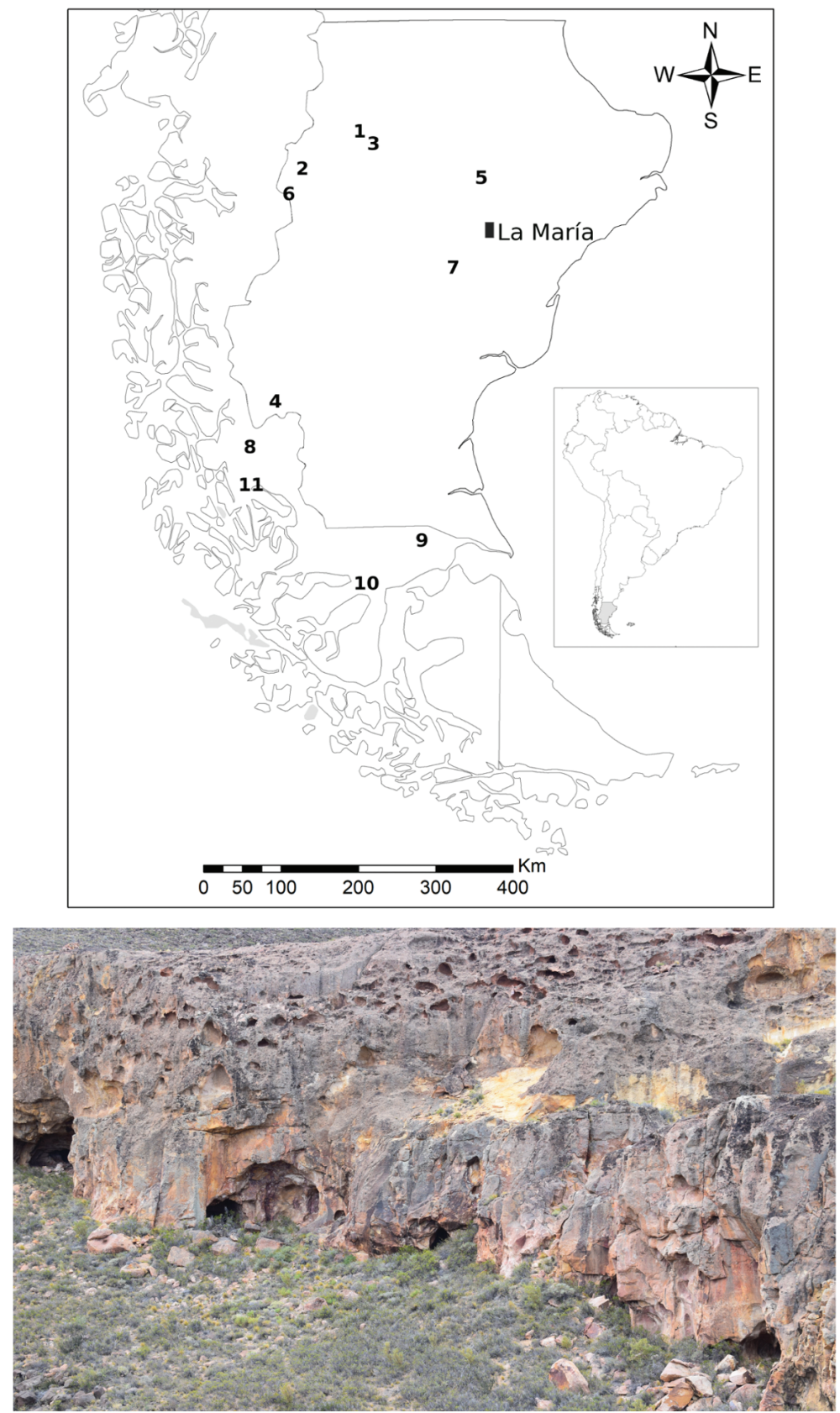

Figura 1. Arriba: localización de La María y otros sitios con análisis composicional. 1: Cueva de las Manos; 2: Cerro de los Indios; 3: Alero Cárdenas; 4: Chorrillo Malo; 5: Cueva Maripe; 6: CCP5; 7: Viuda Quenzana; 8: Laguna Sarmiento 1; 9: Oosin Aike; 10: Cueva Leona; 11: Dos Herraduras, Laguna Sofía 1 y Cueva de Los Niños. Abajo. Panorámica de La María Quebrada. Se observa la boca de las cuevas. Foto: R. González Dubox. 


\section{ARQUEOLOGÍA EN LAS CUEVAS DE LA MARÍA}

Las investigaciones arqueológicas en La María se iniciaron a fines de la década de 1970, cuando Cardich recorrió la localidad y enumeró algunas cuevas pintadas (Cardich, 1979). Franchomme (1991) amplió el relevamiento, fotografió y describió las pinturas halladas, en especial en dos cañadones principales: La María Bajo y La María Quebrada (LMQ, Figura 1).

A mediados de la década de 1990, Paunero inicia sus investigaciones en La María. En el marco de dicho proyecto hemos realizado un relevamiento sistemático que nos permitió reconocer hasta el momento 77 abrigos pintados en distintos sectores de la localidad. De este modo, La María presenta la densidad de pinturas rupestres más elevada de la región (Paunero et al., 2005). La mayor concentración de cuevas pintadas se localiza en LMQ; identificamos 29 sitios con un total de 151 paneles y 1562 motivos pintados allí (R. González Dubox, comunicación personal, junio de 2019). Por su parte, La María Bajo tiene 11 abrigos pintados; la cuantificación de paneles y motivos está en proceso.

La mayor parte de las cuevas pintadas en La María se dan en afloramientos de ignimbritas riolíticas de edad jurásica de la formación Chon Aike (Panza, 1994). Su composición, según estudios por difracción de rayos $X(D R X)$ y análisis de roca total realizados por SEM-EDS, consiste principalmente en cuarzo, sanidina y biotita dentro de una masa felsítica. Los minerales accesorios más comunes incluyen magnetitas titaníferas, ilmenita, calcita, rutilo y circón (Domínguez y Murray, 1997). Es importante resaltar que, como producto de la alteración de las ignimbritas y tobas, abundan en La María caolín y tierras de color que constituyen fuentes potenciales de excelente calidad para la obtención de pigmentos blancos, rojos y amarillos (Paunero et al., 2005).

Paunero y colaboradores (2005) propusieron, para las pinturas de la localidad, una probable sucesión de tres grupos o momentos estilísticos con características y marcos temporales diferentes. El grupo 1 incluye negativos de manos, escenas naturalistas y motivos compuestos por puntos y líneas, pintados con rojos, amarillos y negros. Se lo asigna cronológicamente a la transición Pleistoceno/ Holoceno, entre ca. 11.500 y 7500 años ${ }^{14} \mathrm{C}$ AP. El grupo 2 presenta motivos abstractos y representativos, e incluye guanacos, círculos concéntricos, óvalos y negativos de manos, con gran desarrollo de la policromía. Se reconocieron para este grupo prácticas de mantenimiento de las pinturas, así como la resignificación e incorporación de manifestaciones anteriores para la elaboración de nuevos motivos. Cronológicamente está enmarcado entre los 7500 y 3500 años ${ }^{14} \mathrm{C}$ AP. En el grupo 3, los motivos son geométricos esquemáticos, rectilíneos y en zigzag. Se mantienen los negativos de manos y se desarrolla el estilo de pisadas, con predominio de los colores claros. Este grupo sería del Holoceno tardío, desde 3500 años ${ }^{14} \mathrm{C}$ AP en adelante (Paunero et al., 2005).

Por otra parte, las excavaciones muestran que la localidad estuvo habitada desde hace 11.000 años ${ }^{14} \mathrm{C}$ AP (Paunero et al., 2005). A partir de ese momento se sucedieron ocupaciones cazadoras-recolectoras hasta épocas recientes. Estas sociedades emplearon recursos líticos locales para confeccionar su instrumental aprovechando las abundantes fuentes de rocas de excelente calidad (Skarbun, 2015). El proceso de manufactura incluyó el tratamiento térmico de estas litologías para modificar sus propiedades para la talla (Frank, 2012). También utilizaron, en baja proporción, obsidiana procedente de Pampa del Asador (fuente localizada a ca. 135 $\mathrm{km}$ al oeste). El uso de obsidiana aumenta a partir del Holoceno medio, lo cual indica una intensificación de los lazos con los grupos que habitaban el ecotono bosque-estepa, o bien una ampliación del rango de movilidad para incluir estos espacios de manera más frecuente (Cueto et al., 2018).

\section{LOS ESTUDIOS COMPOSICIONALES EN PATAGONIA MERIDIONAL}

Los estudios sobre la composición físico-química de las pinturas rupestres para la Patagonia meridional (tanto argentina como chilena y considerando como límite norte el río Deseado) comenzaron en la década de 1970. Aunque existen varios trabajos sobre el tema, es escasa la cantidad de muestras analizadas en relación con el número de sitios y motivos relevados. En estos trabajos se aplicó principalmente DRX para analizar la fracción inorgánica cristalina de las pinturas; aunque en menor medida, también se emplearon otras técnicas como SEM-EDS, espectroscopia infrarroja por transformada de Fourier (FTIR), fluorescencia de rayos X y ER (Iníguez y Gradin, 1977; Barbosa y Rial, 1983-1985; Barbosa y Gradin, 1987; Belardi et al., 2000; Wainwright et al., 2002; Sepúlveda, 2011; Carden et al., 2014; Brook et al., 2018). Así, para comprender mejor aspectos relativos 
a los procesos de producción de las pinturas, impera continuar con los análisis incorporando más muestras que incluyan diferentes colores y motivos, empleando distintas vías metodológicas y técnicas analíticas. El abordaje microestratigráfico y multianalítico que presentamos pretende avanzar en ese camino, que hasta el momento tiene un desarrollo relativo en la región.

Los estudios evidenciaron que los colores rojos y amarillos están compuestos por pigmentos ricos en hierro en distintos estados de oxidación. Según Iníguez y Gradin (1977), una figura de un antropomorfo y un guanaco amarillos de Cueva de las Manos (CM) fueron realizados con natrojarosita (un sulfato de sodio y hierro hidratado), mientras que Wainwright et al. (2002) sugieren que las pinturas amarillas de CM y de Cerro de los Indios $(\mathrm{Cl})$ están compuestas por goethita. Barbosa y Gradin (1987) afirman, en base al análisis de pigmentos recuperados en estratigrafía en Alero Cárdenas, que los colores ocres y amarillos están compuestos por jaroisita y goethita respectivamente. Estos tonos se corresponderían a compuestos con limonita en Chorrillo Malo (Belardi et al., 2000). En tanto, en un estarcido amarillo de Cueva Maripe se registró lepidocrocita y hematita (Carden et al., 2014).

Respecto de los rojos, se ha informado la presencia de hematita en pinturas de CM (Iñíguez y Gradin, 1977; Wainwright et al., 2002). Un antropomorfo de dicho sitio contendría hematita y maghemita como cromóforos (Iñíguez y Gradin, 1977). Esta combinación se ha reportado para manchas y guanacos rojos de CCP5 (Barbosa y Rial, 1983-1985; Aschero, 1985), así como en pigmentos recuperados en las excavaciones de Alero Cárdenas y Chorrillo Malo (Barbosa y Gradin, 1987; Belardi et al., 2000). Por su parte, una pintura roja no descripta de $\mathrm{Cl}$ contenía únicamente maghemita (Wainwright et al., 2002). Carden et al. (2014) analizan líneas de puntos y fondos pintados rojos de Cueva Maripe que estarían compuestos por hematita, que también constituye el cromóforo de un motivo de puntos y un negativo de mano de Viuda Quenzana (Brook et al., 2018). En sitios de la Patagonia chilena (Dos Herraduras, Laguna Sofía 1, Laguna Sarmiento 1, Cueva de Los Niños y Oosin Aike), las pinturas rojas fueron producidas por aluminosilicatos asociados a óxidos de hierro (Sepúlveda, 2011). También se ha propuesto que los tonos rojos fueron obtenidos mediante el tratamiento térmico de pigmentos ocres o amarillos (Aschero, 1985; Barbosa y Gradin, 1987; Belardi et al., 2000).
Los análisis de pinturas blancas y negras son aún más restringidos. En CM, un negativo de mano blanco habría sido confeccionado con illita, mientras que en un negativo de mano negro, el cromóforo no fue detectado (Iñíguez y Gradin, 1977). Tanto para CM como para $\mathrm{Cl}$, Wainwright et al. (2002) afirman que las pinturas negras fueron confeccionadas con óxido de manganeso (pirolusita). Los análisis realizados para pinturas blancas por Sepúlveda (2011) indican el uso de una arcilla blanca (Laguna Sarmiento 1), o bien una mezcla de un aluminosilicato con hueso calcinado (Oosin Aike). Por su parte, un trazo negro de Cueva Leona estaría compuesto por aluminosilicatos mezclados con un componente a base de carbón que, según la autora, correspondería a algún tipo de elemento quemado -quizás hueso- (Sepúlveda, 2011).

En estos análisis, además de los elementos y compuestos químicos que pueden asociarse con los cromóforos, también se detectaron otros componentes. Muchos fueron interpretados como minerales accesorios del pigmento y/o como componentes de la roca soporte. Entre ellos destacan el cuarzo, los feldespatos, la caolinita y la mica. Asimismo, en muchas pinturas se identificó yeso, que en ocasiones fue interpretado como un aditivo o carga (Iñíguez y Gradin, 1977; Aschero, 1985; Barbosa y Gradin, 1987; Belardi et al., 2000). Se propuso que el yeso habría sido tratado térmicamente en forma previa a su incorporación a la mezcla pigmentaria (Iñíguez y Gradin, 1977; Barbosa y Gradin, 1987), hecho que estaría corroborado por la presencia de basanita (yeso hemidrato) (Barbosa y Gradin, 1987). Sin embargo, también se ha discutido si la presencia de yeso puede deberse a causas naturales como, por ejemplo, la formación de productos depositados por la acción del agua (Sepúlveda, 2011; Carden et al., 2014).

Finalmente, algunos análisis evidenciaron la presencia de oxalatos de calcio. Estos habrían sido generados por la acción de microorganismos -hongos o líquenes-, o bien podrían ser componentes originales de la pintura (Barbosa y Gradin, 1987; Wainwright et al., 2002; Brook et al., 2018).

\section{METODOLOGÍA Y TÉCNICAS DE ANÁLISIS}

Un inconveniente a la hora de interpretar los resultados de los estudios físico-químicos realizados sobre pinturas rupestres refiere a la correcta diferenciación entre los compuestos correspondientes a las 
pinturas propiamente dichas, a la roca soporte y a posibles depósitos naturales y/o antrópicos previos y/o posteriores a aquellas. A veces esta tarea no es factible dado que la misma técnica de análisis, por ejemplo DRX, requiere pulverizar las muestras para su estudio. No obstante, en otras ocasiones, el análisis de muestras molidas o en bulk responde a una decisión de carácter metodológico y no técnico. Más allá de lo valioso de estas aproximaciones, es necesaria una alternativa metodológica que permita identificar la complejidad de capas y compuestos que conforman y se vinculan a las pinturas. Por ello, en este trabajo empleamos una metodología microestratigráfica combinada con análisis químicos elementales y moleculares mediante dos técnicas: mapeos por SEM-EDS y análisis puntuales por ER. Esta aproximación permite observar y caracterizar en detalle cada una de las capas que componen las estratigrafías, y brinda datos útiles para comprender los materiales utilizados en la confección de las figuras, posibles eventos de repintado y procesos naturales que afectan a los motivos (como formaciones de yeso o de oxalatos de calcio), entre otros aspectos (Gomes et al., 2013; Chalmin et al., 2017).

Para realizar dichos estudios, las muestras fueron tomadas de los paneles siguiendo un protocolo específico, descripto en detalle en otros trabajos (Marte et al., 2014; Tascon et al., 2016). De manera resumida, las muestras - de una superficie aproximada de $1 \mathrm{~mm}^{2}$ - fueron extraídas utilizando bisturí y lupa, y posteriormente almacenadas en tubos plásticos Eppendorf de 1,5 ml. En laboratorio, fueron fotografiadas (anverso y reverso) y luego incluidas en una resina acrílica (Subiton ${ }^{\odot}$ ). Después fueron pulidas con lijas de diferentes granulometrías hasta dejar expuesto un corte transversal que exhibe, mediante la observación en el microscopio óptico, las distintas capas desde la roca base hasta las pinturas o las capas posdepositacionales. Para el registro fotográfico se empleó un microscopio trinocular de polarización, con óptica corregida a infinito de la marca Leica modelo MC 170 HD (full high definition 1080P), para trabajos en luz transmitida y luz incidente con un sistema de iluminación de 35 watios halógenos, revólver para cuatro objetivos e índice de campo visual de $20 \mathrm{~mm}$.

Para el análisis mediante ER se utilizó un espectrómetro Lab RAM HR UV-Vis-NIR (Horiba Jobin Yvon) equipado con doble monocromador y detector CCD (charge coupled device) de resolución espectral de 1,2 $\mathrm{cm}^{-1}$. Se utilizaron las líneas 514,5 nm de un láser de argón y de 632,8 nm de un láser de He-Ne como fuentes excitadoras. La potencia del láser sobre la muestra se mantuvo por debajo de 0,2 $\mathrm{mW}$ para evitar el calentamiento y/o deterioro de la muestra. El microscopio acoplado al espectrómetro (objetivos x10, x50, x100) permite concentrar el haz en una región de estudio de aproximadamente 50 $\mu \mathrm{m} 2$ para un aumento $\times 100$. El tiempo de adquisición y el número de espectros promediados fue ajustado en cada punto; en general fue del orden de 120 segundos de adquisición y cuatro promedios para cada espectro.

La composición elemental fue analizada mediante un microscopio de barrido electrónico SEM-EDS FEI QUANTA 200 (FEI, Oregón, EE.UU.). Para la imagen elemental fue empleado un detector BSD con acelerador de voltaje de $20 \mathrm{kV}$ y corriente de $1,1 \mathrm{nA}$; la distancia de trabajo fue de $10 \mathrm{~mm}$. Las muestras fueron metalizadas con oro (muestras \#21, $\# 24$, \#29, \#47 y \#87) o grafito (muestras \#1, \#3, \#03, \#83 y \#50).

Una de las dificultades en el caso de La María fue la selección de pinturas que se habrían de muestrear, dada la cantidad de motivos, superposiciones y colores involucrados. Se diseñó un plan de muestreo que intentó abordar la mayor diversidad posible contemplando las siguientes variables: color, superposición, grupo estilístico, motivo, técnica de pintado, estado de conservación, tipo de soporte y localización intrasitio de la pintura. Se extrajeron 130 muestras de pinturas y roca soporte. En este artículo presentamos los primeros resultados alcanzados a partir del estudio de 17 muestras. Se analizaron 17 motivos (algunos de los cuales fueron analizados en más de una muestra) y tres soportes próximos a los motivos pero sin evidencias de pinturas. Las muestras proceden de los sectores LMQ (sitios A, H, J/Del Felino, Cueva Larga, La Mesada, Cueva Túnel y La Cocina) y La María Bajo (sitio II). Para este trabajo inicial, se seleccionaron muestras que cubrieran los grandes conjuntos tonales reconocidos en los motivos de la localidad: amarillos, rojos, blancos y negros, procurando incluir muestras con una sola capa de pintura y otras en las que se observaran superposiciones. Debido a la naturaleza microestratigráfica de nuestra metodología, el muestreo de superposiciones permite contar con más de un motivo por muestra (por ejemplo, una mano blanca superpuesta con un motivo de puntos rojos en la muestra \#24). Por ello, la cantidad de estratos sobre la que obtuvimos resultados composicionales $(n=24)$ es superior a la cantidad de muestras analizada $(\mathrm{n}=17)$. Todas las muestras 
fueron fotografiadas en microscopio óptico y analizadas por Raman. Diez de ellas también fueron estudiadas por SEM-EDS.

Para facilitar la interpretación de los datos, exponemos los resultados de acuerdo con el color analizado. En primera instancia, se describen los datos resultantes del estudio de muestras de roca soporte, como línea de base para interpretar los resultados.

\section{RESULTADOS}

\section{Roca soporte}

Se analizaron mediante ER tres muestras de roca soporte sin rastros de pinturas (Tabla 1) tomadas de los sitios $\mathrm{H}$ y J/Del Felino (LMQ, formación Chon Aike). La muestra \#87 exhibe las bandas típicas de la sanidina $\left((\mathrm{KNa})(\mathrm{SiAl})_{4} \mathrm{O}_{8}\right)$ y de la anatasa $\left(\mathrm{TiO}_{2}\right)$, sin detectarse formaciones naturales de oxalatos de calcio u otras sales como yeso. Estudios mediante SEM-EDS de \#87 permiten advertir concentraciones importantes de los elementos de dichos compuestos $(\mathrm{K}, \mathrm{Al}, \mathrm{Si})$, pero también exponen una capa superior rica en calcio (Figura 2). Aunque este sector no fue analizado por ER, probablemente se trate de un depósito de calcita o de algún oxalato de calcio. La ausencia en este estrato de azufre descartaría la presencia de sulfato de calcio.

Otra muestra (\#84) exhibe las bandas Raman de un tipo de óxido de hierro comúnmente denominado burnt sienna en los estudios arqueométricos (Bikiaris et al., 1999). Como veremos, este compuesto suele ser interpretado como el producto del tratamiento térmico de tierras ricas en óxido de hierro. Su hallazgo en la roca soporte sin pinturas visibles plantea diversas hipótesis sobre su origen, pasible de ser interpretado como parte de la geología de la zona, producto del efecto de fuegos

\begin{tabular}{|l|l|l|l|}
\hline Sitio & $\mathbf{N}^{\circ}$ de muestra & \multicolumn{1}{|c|}{ SEM-EDS } & \multicolumn{1}{c|}{ Raman } \\
\hline \multirow{3}{*}{ J/Del Felino } & $\# 84$ & - & Burnt sienna \\
\cline { 2 - 4 } & $\# 87$ & $\begin{array}{l}\mathrm{Al}, \mathrm{C}, \mathrm{Ca}, \mathrm{Fe}, \mathrm{K}, \mathrm{Mg}, \\
\mathrm{Na}, \mathrm{O}, \mathrm{S}, \mathrm{Si}\end{array}$ & Anatasa y sanidina \\
\hline $\mathrm{H}$ & $\begin{array}{l}\# 90 \\
\mathrm{yyy}\end{array}$ & - & Anatasa, goethita y yeso \\
\hline
\end{tabular}

Tabla 1. Resultados obtenidos en muestras de roca soporte. próximos a la roca o hasta como relicto de pinturas deterioradas y no observadas durante la toma de muestras. En \#84 tampoco advertimos las señales típicas de los sulfatos u oxalatos de calcio. Por último, en \#90
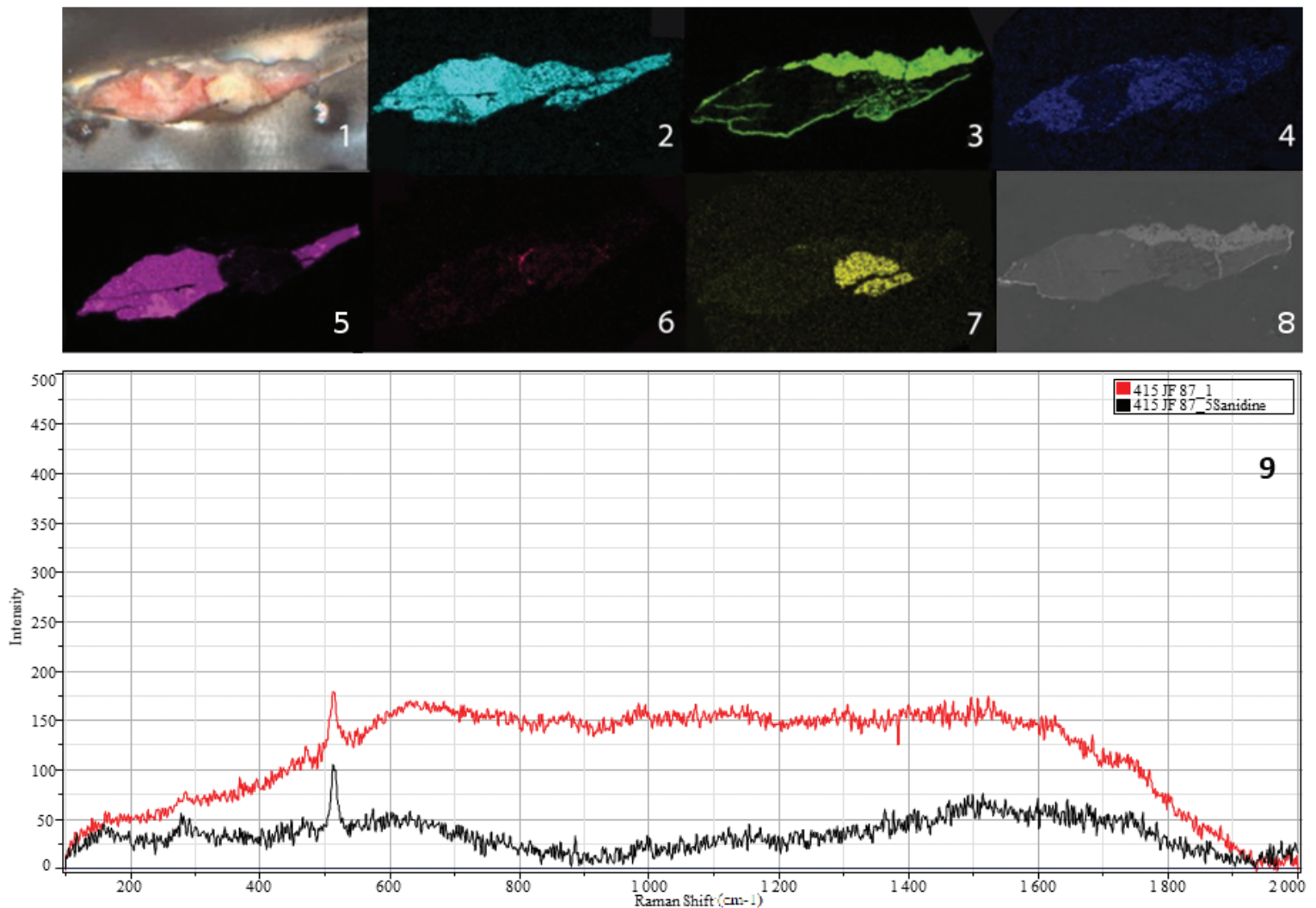

Figura 2. 1: Micrografía \#87 100X, Mapeos SEM-EDS 2: aluminio, 3: calcio, 4: magnesio, 6: hierro, 7: azufre, 8: silicio. 5: imagen obtenida mediante electrones retrodispersados 9: espectro Raman de \#87; en rojo se observa la presencia de sanidina, y en negro, el espectro patrón del mismo mineral. 
fueron identificados los compuestos anatasa $\left(\mathrm{TiO}_{2}\right)$, goethita $(\alpha-\mathrm{FeO}(\mathrm{OH}))$ y yeso $\left(\mathrm{CaSO}_{4} \cdot 2 \mathrm{H}_{2} \mathrm{O}\right)$; los dos primeros probablemente asociados a la constitución de la roca soporte, y el yeso, vinculado a una formación natural posterior. Algunos de estos compuestos también fueron hallados en pinturas de la localidad, por lo que debemos ser cautos para no confundir los compuestos propios de las pinturas con aquellos de las rocas soporte.

\section{Pinturas amarillas}

Se analizaron con ER y SEM-EDS dos muestras correspondientes a sendos motivos amarillos: un óvalo y un negativo de mano (Tabla 2, Figura 3). En una de las muestras, el estrato amarillo se superpone con uno blanco y otro rojo.

Los estudios elementales en ambas muestras expusieron concentraciones de Fe, Si y $\mathrm{Al}$ en el estrato pigmentario. Por su parte, los análisis por ER detectaron las bandas asignables a la goethita (Figura 3),

\begin{tabular}{|l|l|l|l|l|c|}
\hline Motivo & Sitio & $\mathbf{N}^{\circ}$ de muestra & SEM-EDS & Raman & GE \\
\hline Óvalos & $\begin{array}{l}\text { J/Del } \\
\text { Felino }\end{array}$ & $\# 3$ & $\begin{array}{l}\mathrm{C}, \mathrm{O}, \mathrm{Fe}, \mathrm{Al}, \mathrm{Si}, \mathrm{S}, \\
\mathrm{Ca}, \mathrm{Cl}, \mathrm{Mg}, \mathrm{Na}, \mathrm{K}\end{array}$ & Hidróxido de hierro & 3 \\
\hline Negativo de mano & La Cocina & $\# 29$ & $\mathrm{Al}, \mathrm{Fe}, \mathrm{Si}$ & Goethita, wedellita, cuarzo & 3 \\
\hline
\end{tabular}

Tabla 2. Resultados obtenidos en muestras de pinturas de tonos amarillos. GE: grupo estilístico.
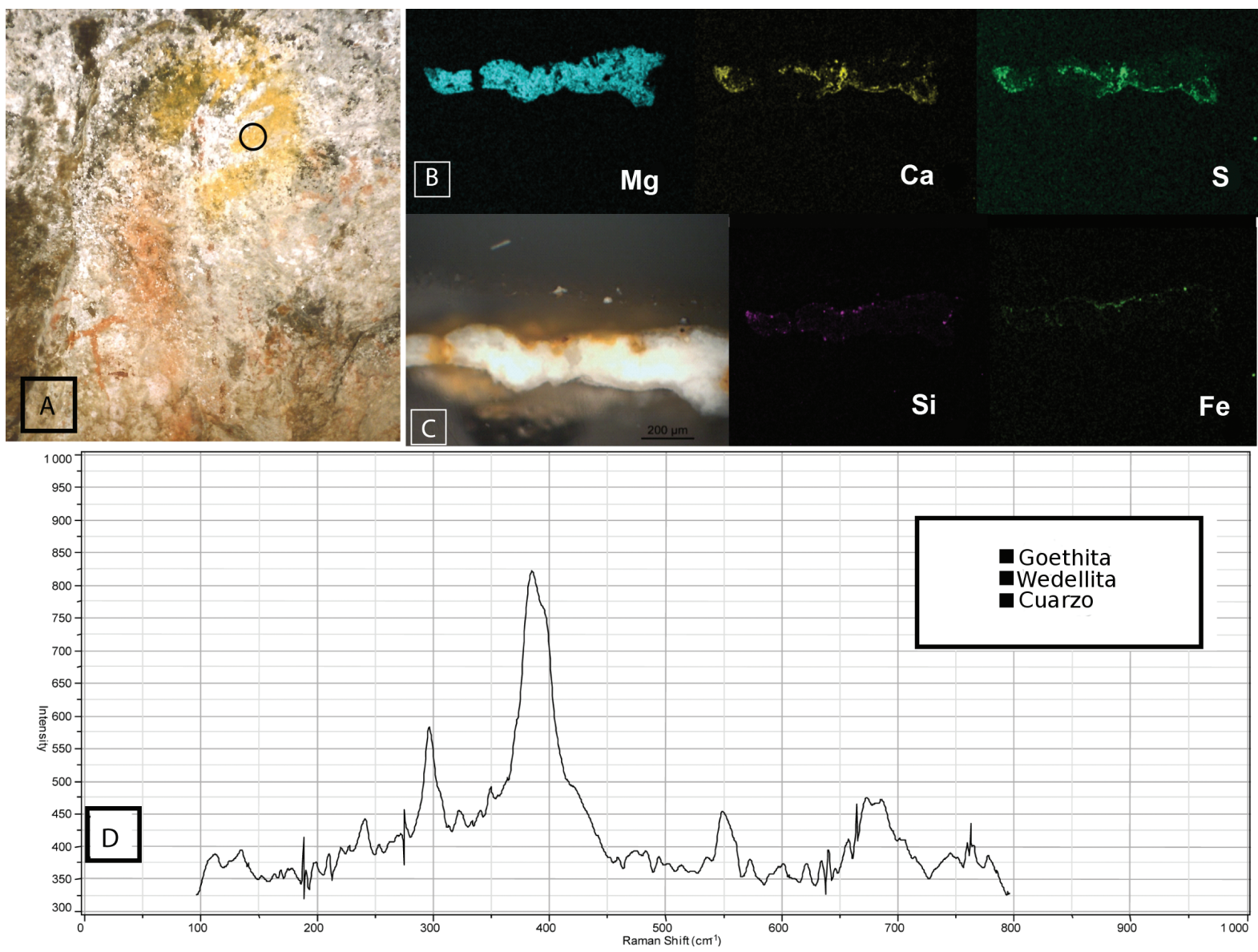

Figura 3. A. Motivo muestreado (con un círculo se marca el lugar de extracción de \#29). B. Mapeos SEM-EDS. C. Microestratigrafía \#29, D. Espectro Raman de \#29, que muestra goethita, wedellita y cuarzo. 
hierro, según se observa en los resultados obtenidos por ER.

La observación de las microestratigrafías y la comparación detallada de los resultados químicos permite advertir pequeñas diferencias entre las pinturas rojas, que nos inclinan a agruparlas en tres conjuntos:

- Conjunto 1 (\#23 y \#24): estas pinturas presentan las bandas Raman asignables al burnt sienna, un tipo de tierra rojiza tostada que, en términos químicos, se puede describir como una mezcla entre hematita y magnetita $\left(\mathrm{Fe}_{2} \mathrm{O}_{3}\right.$ $+\mathrm{Fe}_{3} \mathrm{O}_{4}$ ). También fueron detectados cuarzo $\left(\mathrm{SiO}_{2}\right)$ y anhidrita, un sulfato de calcio deshidratado $\left(\mathrm{CaSO}_{4}\right)$, quizás usado como carga en las pinturas. Algunas pequeñas partículas negras inmersas en el estrato rojizo arrojaron las seña- les de la magnetita (Figura 4). También fueron localizados oxalatos de calcio, en forma de wedellita $\left(\mathrm{CaC}_{2} \mathrm{O}_{4} \cdot 2 \mathrm{H}_{2} \mathrm{O}\right)$, en la capa pictórica.

- Conjunto 2 (\#1,\#3, \#47 y \#99): el análisis expuso señales de óxidos de hierro, en algunos casos como hematita y en otros como burnt sienna. Salvo en \#99, en el resto también fueron detectados oxalatos de calcio, en algunos casos como whewellita $\left(\mathrm{CaC}_{2} \mathrm{O}_{4} \cdot \mathrm{H}_{2} \mathrm{O}\right)$ y en otros como wedellita. A diferencia del conjunto anterior, no se identificaron sulfatos de calcio.

- Conjunto 3 (\#21, \#50 y \#51): en estas pinturas se detectaron hematita y yeso (Figura 5). A diferencia del primer conjunto, este grupo no exhibe señales del burnt sienna. El sulfato de calcio fue hallado en su forma dihidrato (yeso). También fueron localizados, salvo en \#21, oxalatos de calcio (wedellita).

\begin{tabular}{|c|c|c|c|c|c|}
\hline Motivo & Sitio & $\begin{array}{c}\mathrm{N}^{\circ} \text { de } \\
\text { muestra }\end{array}$ & SEM-EDS & Raman & GE \\
\hline \multirow[t]{2}{*}{ Puntos } & \multirow[t]{2}{*}{ Larga } & $\# 23$ & - & Burnt sienna, anhidrita & \multirow[b]{2}{*}{$2 / 3 ?$} \\
\hline & & $\# 24$ & $\begin{array}{l}\mathrm{Al}, \mathrm{Ca}, \mathrm{Fe}, \mathrm{K}, \mathrm{S}, \mathrm{Si}, \\
\mathrm{Ti}\end{array}$ & $\begin{array}{l}\text { Burnt sienna, anhidrita, } \\
\text { wedellita, magnetita (en } \\
\text { partículas negras). }\end{array}$ & \\
\hline \multirow[t]{2}{*}{ Guanaco } & \multirow[t]{2}{*}{ J/Del Felino } & $\# 1$ & $\begin{array}{l}\mathrm{C}, \mathrm{O}, \mathrm{Fe}, \mathrm{Na}, \mathrm{Mg} \text {, } \\
\mathrm{Al}, \mathrm{Si}, \mathrm{P}, \mathrm{S}, \mathrm{Cl}, \mathrm{Ca} .\end{array}$ & $\begin{array}{l}\text { Hematita, whewellita, carbón } \\
\text { (en partículas negras) }\end{array}$ & \multirow{2}{*}{2} \\
\hline & & $\# 3$ & $\begin{array}{l}\mathrm{C}, \mathrm{O}, \mathrm{Fe}, \mathrm{Mg}, \mathrm{Al} \text {, } \\
\mathrm{Si}, \mathrm{K}, \mathrm{Ca}\end{array}$ & Hematita, whewellita & \\
\hline Estarcido & A & $\# 47$ & $\begin{array}{l}\mathrm{Al}, \mathrm{Ca}, \mathrm{Fe}, \mathrm{K}, \mathrm{O}, \mathrm{S}, \\
\mathrm{Si}\end{array}$ & Burnt sienna, weddellita & $2 / 3 ?$ \\
\hline Línea indet. & Cueva Túnel & \#99 & - & Hematita, ortoclasa & Indet. \\
\hline $\begin{array}{l}\text { Guanacos } \\
\text { tomando agua }\end{array}$ & $\mathrm{H}$ & $\# 21$ & $\mathrm{Al}, \mathrm{Ca}, \mathrm{Fe}, \mathrm{K}, \mathrm{O}, \mathrm{Si}$ & Hematita, yeso & 2 \\
\hline Puntos & La Mesada & $\# 50$ & $\begin{array}{l}\mathrm{C}, \mathrm{O}, \mathrm{Fe}, \mathrm{Na}, \mathrm{Mg}, \\
\mathrm{Al}, \mathrm{Si}, \mathrm{P}, \mathrm{S}, \mathrm{K}, \mathrm{Ca} .\end{array}$ & Hematita, yeso & 3 \\
\hline $\begin{array}{l}\text { Círculo } \\
\text { compuesto } \\
\text { concéntrico }\end{array}$ & La Mesada & $\# 51$ & - & $\begin{array}{l}\text { Hematita, yeso, wedellita y } \\
\text { anatasa }\end{array}$ & 2 \\
\hline
\end{tabular}

De manera preliminar, estos tres conjuntos podrían interpretarse como mezclas pigmentarias diferentes para obtener colores similares. Este aspecto resulta interesante dado que expone, aun de forma simplificada, la heterogeneidad de las pinturas. En el caso

Tabla 3. Resultados obtenidos en muestras de pinturas de tonos rojos y naranjas. GE: grupo estilístico.
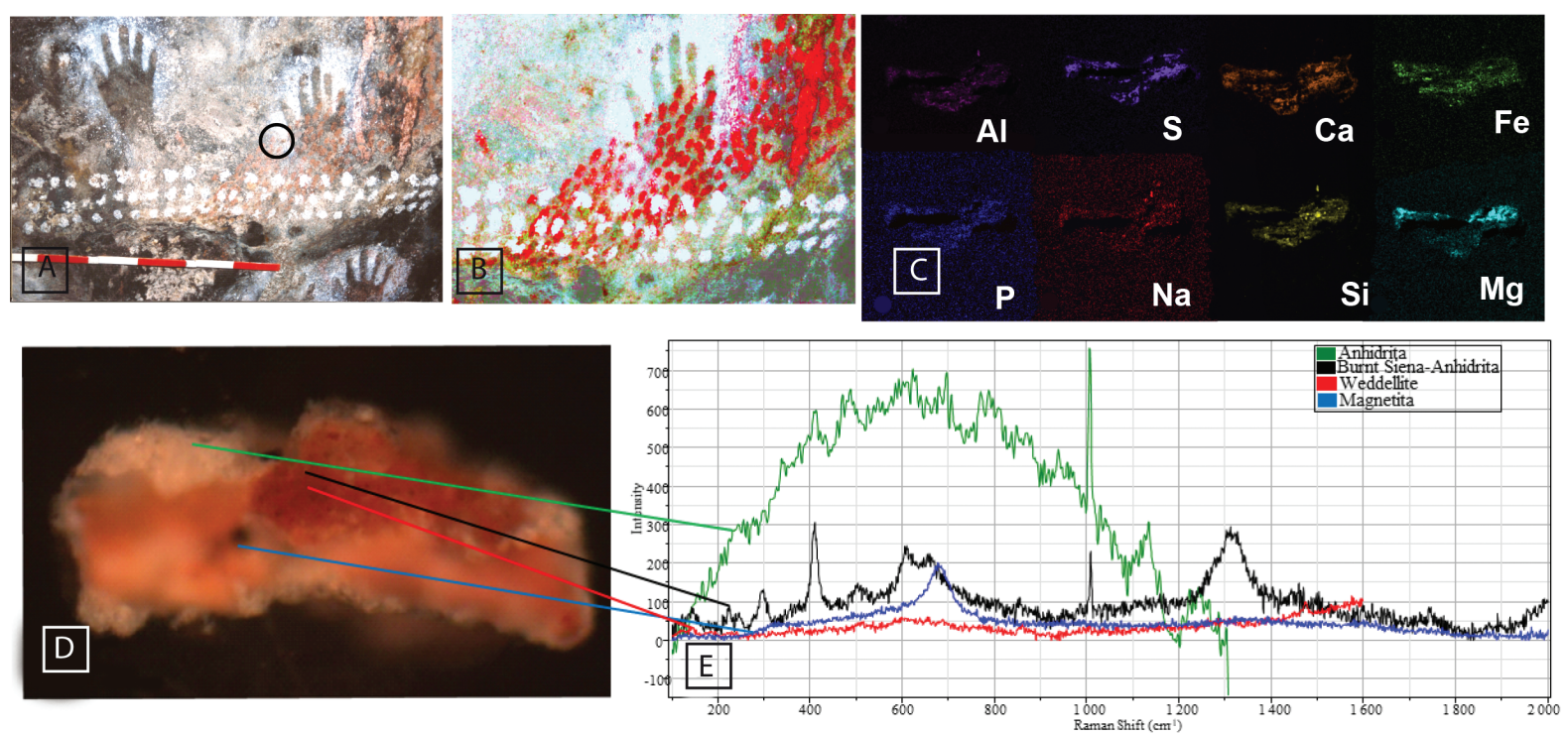

Figura 4. A. Motivo muestreado (en círculo se marca el lugar de extracción de \#24). B. Imagen procesada por DStretch (filtro ywe) que resalta el motivo rojo. C. Mapeos SEM-EDS 1. D. Microestratigrafía \#24 con señalización del punto de adquisición de cada espectro de Raman. E. Espectro Raman de \#24 que muestra anhidrita, burnt sienna, weddellita y magnetita. 

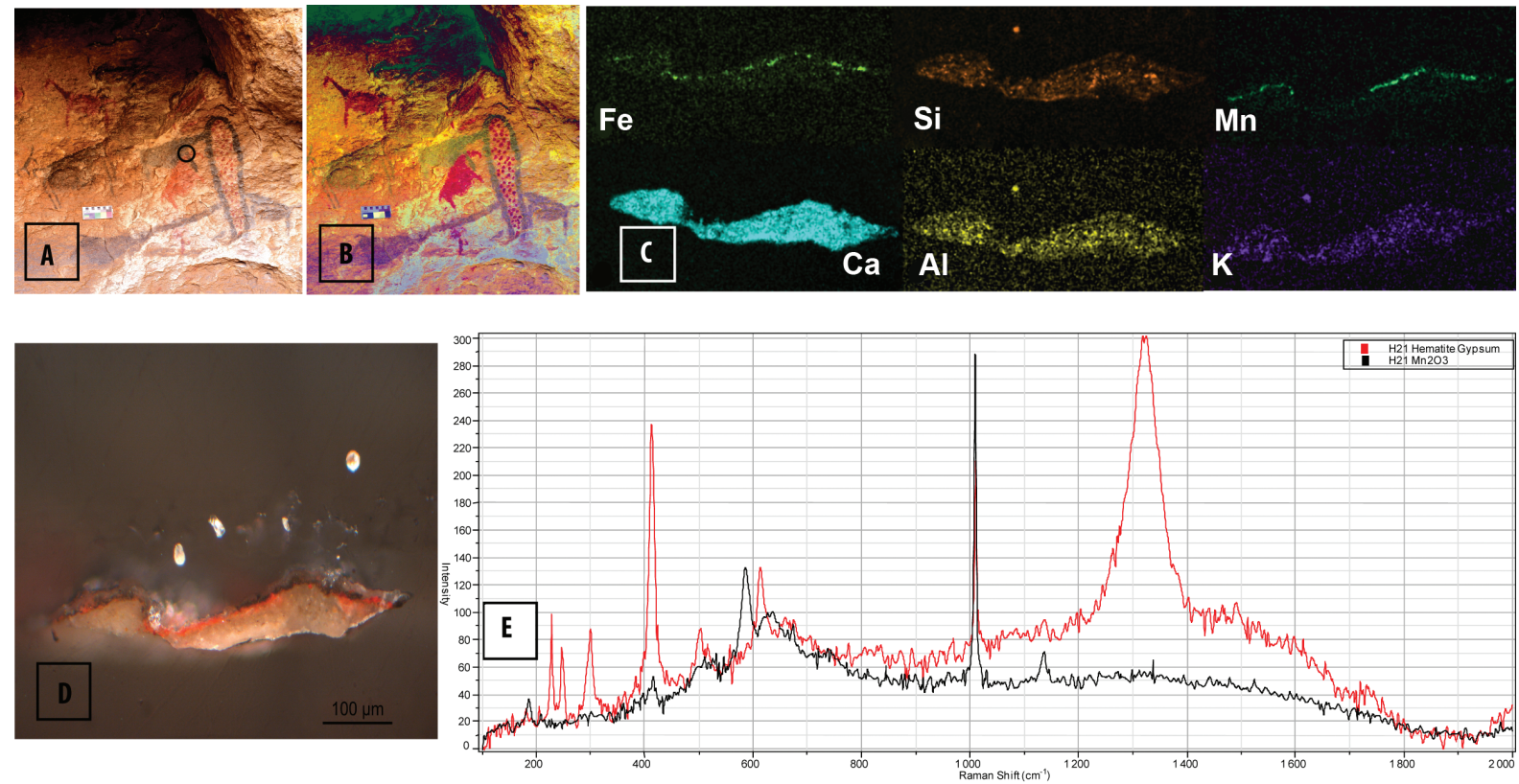

Figura 5. A. Motivo muestreado (en círculo se marca el lugar de extracción de \#21). ). B. Imagen procesada por DStretch (filtro Ids), en la que se resalta el motivo rojo. C. Mapeos SEM-EDS. D. Microestratigrafía \#21. E. Espectro Raman de \#21 que muestra óxido de manganeso, yeso y hematita.

de los rojos, esta heterogeneidad está fundamentada en el uso de distintos tipos de óxido de hierro (burnt sienna o hematita) y por la presencia diferencial de sulfatos de calcio en distintos estados de hidratación (yeso o anhidrita). En tal sentido, es importante hacer algunos comentarios sobre estos hallazgos.

Como vimos, varios de los análisis por ER indicaron las bandas correspondientes al burnt sienna $\left(224,293,410,498,612,659 \mathrm{~cm}^{-1}\right)$ (Bikiaris et al., 1999). Estos datos inmediatamente recordaron las hipótesis sobre el tratamiento térmico de pigmentos esbozadas para la región (Aschero, 1985; Barbosa y Gradin, 1987; Belardi et al., 2000). Si bien la similitud con algunos espectros Raman expuestos en otros artículos (Gomes et al., 2013) nos inclinó a proponer esta interpretación, el hallazgo de caolinita $\left(\mathrm{Al}_{2} \mathrm{Si}_{2} \mathrm{O}_{5}(\mathrm{OH})_{4}\right)$ en tierras rojas de La María nos hizo dudar de si dichas bandas podrían corresponder a este compuesto. En ese sentido, Bikiaris et al. (1999) exponen los resultados de lo que denominan red ochre, una tierra arcillosa rojiza que presenta las señales típicas de la hematita sumadas a las bandas de la caolinita, principalmente la correspondiente a los $658 \mathrm{~cm}^{-1}$, que genera un espectro Raman muy similar al atribuido al burnt sienna. Sin embargo, los análisis ER realizados en las muestras de pinturas de La María donde detectamos burnt sienna no localizaron las señales de la caolinita (3621 y $\left.3697 \mathrm{~cm}^{-1}\right)$, por lo que descartamos esta hipótesis. A pesar de ello, no es posible atribuir directamente este compuesto a la aplicación de tratamiento térmico dado que, como vimos más arriba, también fue localizado en la roca soporte sin pinturas. Por lo tanto, el origen del burnt sienna hallado en las pinturas será un punto a profundizar en futuros trabajos.

El segundo aspecto importante que se desprende del estudio de las pinturas rojas se vincula con el hallazgo de sulfatos de calcio, en ocasiones en forma hidratada (yeso), pero también deshidratados (anhidrita). El hallazgo de anhidrita abre nuevos interrogantes sobre la posible transformación térmica de este compuesto a partir de yeso natural, hecho que ya fuera sugerido por Iñíguez y Gradin (1977) y Barbosa y Gradin (1987). De una u otra forma es probable que los sulfatos de calcio hayan sido utilizados como carga de algunas mezclas pigmentarias rojas, puesto que fueron detectados en las mismas capas de donde obtuvimos las señales de los óxidos de hierro.

\section{Pinturas blancas}

Se examinaron siete muestras de pinturas blancas correspondientes a seis motivos: dos negativos de mano, un círculo concéntrico, un óvalo, un motivo de puntos y otro de líneas paralelas (Tabla 4, Figura 4). En cuatro muestras, los estratos blancos se superponen con capas rojas.

Los resultados obtenidos para \#83 permitieron detectar las bandas Raman características de la anatasa $\left(\mathrm{TiO}_{2}\right)$. Estudios elementales de la misma 
pintura arrojaron altas concentraciones de $\mathrm{Al}$ y $\mathrm{Si}$ en el estrato blanco, lo cual podría indicar la presencia de alguna arcilla, no detectada por ER. Otra muestra analizada, extraída del mismo sitio - -j/del Felino- corresponde a un negativo de mano (\#5). En este caso fueron detectadas las bandas espectrales correspondientes al yeso y a la anatasa, sin localizar señales de arcilla. Por el contrario, un tipo de arcilla (caolinita) fue detectada por ER en \#65 junto con las señales de la anhidrita. En \#23 y \#24 se localizaron señales de anhidrita (Figura 4) y, en una de ellas, también de cuarzo.

Como en las pinturas rojas, los resultados obtenidos para las muestras blancas indican diferentes composiciones químicas que podrían asociarse con una heterogeneidad de mezclas pigmentarias de los mismos colores presentes en la localidad. Si bien los resultados mostrarían el uso del sulfato de calcio $y$, posiblemente, de la caolinita como pigmentos, estos no son definitivos y es necesario continuar con los análisis.

Debemos mencionar que en La María abundan los afloramientos de caolinita que han sido explotados de forma comercial. Los análisis realizados sobre rocas blancas de estas canteras arrojan las bandas Raman características de la caolinita. Estudios por FT-IR y DRX de las mismas muestras corroboran la presencia de dicho compuesto. Aunque estos datos no posibilitan asegurar que las pinturas fueran extraídas de esas canteras, sí permiten contextualizarlas en una zona geológica en la cual existe este compuesto, incluso detectado al estudiar sedimentos de otros colores (rojos, amarillos). Cabe destacar que este compuesto puede pasar inadvertido en los análisis por ER, dado que sus bandas características se ubican en el 3621 y $3697 \mathrm{~cm}^{-1}$, un sector del espectro que muchas veces no es explorado.

\section{Pinturas negras}

Fueron analizadas tres muestras correspondientes a dos motivos: una escena de guanacos tomando agua y una línea continua rellena (Tabla 5, Figura 5). En dos de ellas se superponen motivos rojos y negros. Los resultados elementales por SEM-EDS indicaron mayores concentraciones de manganeso $(\mathrm{Mn})$ en los estratos negros de las tres muestras (Figura 5). Además de dicho elemento, se advierten concentraciones de calcio y, en \#47, algo de carbono. Estos datos se condicen parcialmente con los obtenidos mediante ER, los cuales solo detectaron las señales de óxidos de manganeso en dos muestras del mismo motivo ( $\# 21$ y \#22). Sin embargo, en \#47 fueron localizadas las bandas asignables al carbono amorfo, en 1350 y $1601 \mathrm{~cm}^{-1}$. Estas diferencias podrían indicar una combinación de óxidos de manganeso y carbón en algunas mezclas pigmentarias, aunque el número reducido de muestras analizadas no nos permite corroborar la

\begin{tabular}{|c|c|c|c|c|c|}
\hline Motivo & Sitio & $N^{\circ}$ de muestra & SEM-EDS & Raman & GE \\
\hline \multirow[t]{2}{*}{$\begin{array}{l}\text { Óvalos continuos } \\
\text { paralelos }\end{array}$} & \multirow[t]{2}{*}{ J/Del Felino } & $\# 83$ & $\begin{array}{l}\mathrm{O}, \mathrm{Mg}, \mathrm{C}, \mathrm{Al}, \mathrm{Si}, \mathrm{P}, \\
\mathrm{S}, \mathrm{K}, \mathrm{Ca}, \mathrm{Fe}, \mathrm{Na} .\end{array}$ & Anatasa & \multirow[t]{2}{*}{3} \\
\hline & & $\# 3$ & $\mathrm{C}, \mathrm{O}, \mathrm{Ca}, \mathrm{Si}, \mathrm{S}, \mathrm{Cl}$ & $\mathrm{S} / \mathrm{r}$ & \\
\hline $\begin{array}{l}\text { Negativo de mano } \\
\text { con puntos (Mano) }\end{array}$ & J/Del Felino & $\# 5$ & - & Yeso, anatasa & 3 \\
\hline Puntos & Larga & $\# 23$ & - & Anhidrita & 3 \\
\hline Negativo de mano & Larga & $\# 24$ & $\mathrm{Ca}, \mathrm{S}$ & $\begin{array}{l}\text { Anhidrita, } \\
\text { cuarzo }\end{array}$ & 3 \\
\hline $\begin{array}{l}\text { Círculo compuesto } \\
\text { concéntrico }\end{array}$ & La Mesada & $\# 50$ & $\begin{array}{l}\mathrm{C}, \mathrm{O}, \mathrm{Fe}, \mathrm{Na}, \mathrm{Mg}, \mathrm{Al}, \mathrm{P}, \mathrm{S}, \\
\mathrm{Ba}, \mathrm{K}, \mathrm{Ca}, \mathrm{Si}\end{array}$ & $\mathrm{S} / \mathrm{r}$ & 2 \\
\hline Líneas paralelas & II & $\# 65$ & - & $\begin{array}{l}\text { Caolinita, } \\
\text { anhidrita }\end{array}$ & 2 \\
\hline
\end{tabular}

Tabla 4. Resultados obtenidos en muestras de pinturas de tonos blancos. GE: grupo estilístico. S/r: sin resultados.

\begin{tabular}{|l|l|l|l|l|c|}
\hline \multicolumn{1}{|c|}{ Motivo } & Sitio & $\mathbf{N}^{\circ}$ de muestra & \multicolumn{1}{|c|}{ SEM-EDS } & \multicolumn{1}{c|}{ Raman } & GE \\
\hline $\begin{array}{l}\text { Guanacos tomando } \\
\text { agua (Guanaco) }\end{array}$ & $\mathrm{H}$ & $\# 21$ & Mn & $\begin{array}{l}\text { Óxido de manganeso (lambda } \\
\text { MnO + MnFe O) }\end{array}$ & 2 \\
\cline { 2 - 5 } & $\# 22$ & - & $\begin{array}{l}\text { Óxido de manganeso (lambda } \\
\text { Mn O + MnFe O) }\end{array}$ & 2 \\
\hline $\begin{array}{l}\text { Línea continua } \\
\text { rellena }\end{array}$ & $\mathrm{A}$ & $\# 47$ & $\begin{array}{l}\mathrm{C}, \mathrm{Ca}, \mathrm{K}, \mathrm{Mn}, \\
\mathrm{O}, \mathrm{S}, \mathrm{Si}\end{array}$ & Carbón, anhidrita & 2 \\
\hline
\end{tabular}

Tabla 5. Resultados obtenidos en muestras de pinturas de tonos negros. GE: grupo estilístico. 
hipótesis. En cuanto a la presencia de sulfatos de calcio, algunas bandas Raman detectadas en \#21 permitirían inferir la presencia de yeso, aunque llama la atención su ausencia en $\# 22$, perteneciente al mismo motivo. La presencia de azufre y calcio en los mapeos SEM-EDS del estrato negro de \#47 nos permitió considerar la presencia de sulfatos de calcio, luego confirmada a partir del hallazgo de las señales de la anhidrita.

\section{DISCUSIÓN Y CONCLUSIONES}

Este trabajo constituye el primer abordaje arqueométrico de las pinturas de La María. Los estudios microestratigráficos implementados son una vía novedosa para el estudio de los pigmentos en el arte rupestre de Patagonia meridional. Su combinación con Ios análisis por ER y SEM-EDS ha permitido caracterizar químicamente los estratos pictóricos individuales de cada motivo, sin destruir la muestra y diferenciándolos de la roca soporte.

Los resultados preliminares muestran que la composición de las pinturas es bastante heterogénea, lo cual indicaría que existieron distintas recetas o modos de lograr cada tonalidad. Probablemente se emplearon diferentes variedades de pigmentos para cada color, en ocasiones junto con otros compuestos como sulfatos de calcio (yeso o anhidrita), arcillas (caolinitas) y cuarzo. En el caso de las pinturas de tonalidades rojizas, los estudios ejecutados nos permiten advertir que existen mezclas realizadas con dos tipos de pigmentos basados en óxidos de hierro, hematita y burnt sienna, combinados en algunos casos con yeso o anhidrita. Para los tonos negros se empleó óxido de manganeso; este en algunas ocasiones probablemente fuera mezclado con carbón. Al igual que con los motivos rojos, solo en algunas pinturas negras se detectó yeso. En referencia a los blancos, los resultados parecen indicar la utilización de sulfatos de calcio y, quizás, caolinita como cromóforos. Finalmente, las pinturas amarillas exhiben un único patrón composicional, con el empleo de un hidróxido de hierro (goethita) sin la adición de yeso. Sin embargo, la cantidad de muestras analizada es limitada.

Esta variabilidad de mezclas pigmentarias es consistente con la gran variedad de motivos, colores, técnicas y extensión cronológica del proceso de pintado en La María. La diversidad composicional puede obedecer a diferentes factores. Es probable que se hayan dado distintos eventos de pintado sucedidos en el tiempo. Esto se condice con las hipótesis sobre la gran extensión temporal del proceso de pintado de la localidad. También es posible que existan diferentes formas de preparación relacionadas con distintos tipos de pinturas o técnicas de confección, o incluso que en La María hayan pintado distintas comunidades con diferentes materias primas y formas de preparar las pinturas. Aunque la extensión del artículo y la cantidad de muestras analizada nos impiden profundizar sobre el tema, interesa remarcar que no hemos observado patrones composicionales claros en función del grupo estilístico al que fueron asignados los motivos. Por ejemplo, las pinturas de los grupos 2 y 3 muestran el uso de hematita y el empleo de mezclas con y sin sulfatos de calcio (no se analizaron motivos del grupo 1). En tanto, el burnt sienna fue registrado solo en motivos en los que la asignación a un determinado grupo estilístico es aún dudosa. Estas observaciones son preliminares y realizaremos estudios específicos para abordar estas cuestiones.

Un aspecto necesario de evaluar es la procedencia de las materias primas empleadas. Las características composicionales relevadas en pinturas rojas, amarillas y blancas son, en principio, coherentes con los afloramientos y tierras disponibles localmente. Esto sería análogo al uso de rocas locales que se registró para la manufactura lítica (Skarbun, 2015). Sin embargo, son necesarios estudios más precisos de estos afloramientos a nivel local y regional. En este sentido, el hecho de que el origen geológico de los pigmentos potencialmente empleados sea el mismo que el de la roca soporte plantea un problema de equifinalidad. Por ello, aunque somos cautos en las apreciaciones, deseamos poner en valor la aproximación microestratigráfica, dado que permite deslindar los resultados de los análisis químicos por capa y no en bulk. Por otra parte, es interesante mencionar que no se han registrado aún potenciales fuentes de óxido de manganeso cerca de La María, por lo cual este pigmento sería alóctono. Planteamos como hipótesis preliminar que este tipo de pigmento provendría del sector occidental, dado el vector de movilidad E-W reconocido a partir del análisis de obsidiana (Cueto et al., 2018) y considerando que las pinturas negras en CM y Cl también contenían óxidos de manganeso (Wainwright et al., 2002).

Una comparación inicial de los resultados obtenidos con aquellos disponibles a nivel regional también nos permite delinear algunas conclusiones preliminares. Aunque la presencia de óxidos de hierro en pinturas rojas es acorde con lo registrado 
en el resto de la región (Iñíguez y Gradin, 1977; Barbosa y Rial, 1983-1985; Wainwright et al., 2002; Sepúlveda, 2011; Carden et al., 2014; Brook et al., 2018), observamos que en algunos de los análisis previos los compuestos identificados difieren de los aquí registrados. Esto también indicaría el uso de materias primas distintas en diferentes sitios, aunque surge aquí la incógnita de si las diversas metodologías y técnicas analíticas empleadas afectan el tipo de resultados obtenidos como consecuencia de la alta sensibilidad de algunos equipos para la detección de determinados compuestos y las dificultades para obtener señales de otros. Para obtener los colores blancos, se ha propuesto el empleo de la illita o, incluso, la mezcla de una arcilla blanca con hueso calcinado (Iníguez y Gradin, 1977; Sepúlveda, 2011), compuestos que no hemos hallado en La María. En el caso de los amarillos, si bien el compuesto suele ser la goethita, también se han mencionado otros como la natrojaroisita, la jaroisita, la lepidocrocita y la limonita (Iñíguez y Gradin, 1977; Barbosa y Gradin, 1987; Belardi et al., 2000; Carden et al., 2014). Finalmente, los escasos estudios previos que reportaron análisis de pinturas negras propusieron el uso del óxido de manganeso o de un componente a base de carbón (Wainwright et al., 2002; Sepúlveda, 2011), pero esta es la primera ocasión en la que se propone una potencial mezcla entre ambos. Es decir, la heterogeneidad en la composición de las pinturas se daría tanto a escala local como regional. Entonces, se hace menester implementar un abordaje regional, que amplíe la cantidad de muestras analizadas para los distintos colores y contemple la realización de estudios con técnicas similares y complementarias que brinden información factible de ser comparada de forma segura.

Un último aspecto a reseñar se vincula con el posible tratamiento térmico de algunas mezclas pigmentarias, hipótesis planteada previamente para el arte rupestre patagónico. Recordemos que esta técnica ya fue corroborada para la manufactura de artefactos líticos en La María, lo cual evidencia un manejo controlado del fuego y el conocimiento de sus propiedades tecnológicas (Frank, 2012). Los resultados obtenidos plantean indicios pasibles de ser atribuidos a este procedimiento, aunque también advierten respecto de los límites de dichas interpretaciones. Así, el hallazgo de burnt sienna en pinturas rojas podría ser producto del tratamiento térmico de tierras ricas en óxido de hierro. Sin embargo, su detección en la roca soporte sin pinturas abre interrogantes sobre el origen de este compuesto. De igual modo, resulta llamativo que en las muestras de pinturas en donde detectamos este compuesto también hallamos señales de anhidrita (sulfato de calcio deshidratado), a diferencia de lo que ocurre cuando el pigmento es hematita, siempre asociada al yeso (sulfato de calcio dihidrato). La anhidrita puede obtenerse a partir del calentamiento del yeso o la basanita (sulfato de calcio hemihidratado) a temperaturas mayores a $110{ }^{\circ} \mathrm{C}$ (Prieto-Taboada et al., 2014), aunque también puede presentarse naturalmente. La ausencia de anhidrita en los análisis realizados sobre rocas soporte -donde hallamos yeso-, y su recurrente asociación con burnt sienna podrían ser indicios del tratamiento térmico de las mezclas pigmentarias. Estos aspectos serán profundizados en el futuro.

\section{Agradecimientos}

Agradecemos a la familia Behm, que gentilmente nos recibe y acompaña en La María. A Raúl González Dubox y Fabiana Skarbun. A tres evaluadores por sus valiosas sugerencias. Este trabajo fue financiado por CONICET (PIP0785) y la UNLP (N811).

\section{REFERENCIAS CITADAS}

Aschero, C. (1985). Notas sobre el uso de pigmentos minerales en el sitio CCP-5, provincia de Santa Cruz, Argentina. En C. Aldunate, J. Berenguer y V. Castro (Eds.), Estudios en arte rupestre. Primeras jornadas de arte y arqueologia, el arte rupestre en Chile. Santiago 16 al 19 de agosto de 1983, 13-24. Museo Chileno de Arte Precolombino.

Aschero, C. (1996). ¿Adónde van esos guanacos? En J. Gómez Otero (Ed.), Arqueología. Solo Patagonia (pp. 153-162). Centro Nacional Patagónico (CENPAT).

Barbosa, C. y Gradin, C. (1987). Estudio composicional por difracción de rayos $\mathrm{X}$ de los pigmentos provenientes de la excavación del Alero Cárdenas (provincia de Santa Cruz). Relaciones de la Sociedad Argentina de Antropología, XVII(1), 143-171.

Barbosa, C. y Rial, G. (1983-1985). Análisis mineralógico por difracción de rayos $\mathrm{X}$ de muestras de pinturas del Cerro Casa de Piedra, Sitio CCP5 (Provincia de Santa Cruz, Argentina). Cuadernos del Instituto Nacional de Pensamiento Latinoamericano, 10, 307-311.

Belardi, J. B., Súnico, A. y Puebla, D. (2000). Análisis de pigmentos minerales y sus fuentes potenciales de aprovisionamiento en el área de Lago Roca (Sector 
Chorrillo Malo), Provincia de Santa Cruz (Argentina). Anales del Instituto de la Patagonia, 28, 291-304.

Bikiaris, D., Daniilia, S., Sotiropoulou, S., Katsimbiri, O., Pavlidou, E., Moutsatsou, A. y Chryssoulakis, Y. (1999). Ochre-differentiation through micro-Raman and microFTIR spectroscopies: application on wall paintings at Meteora and Mount Athos, Greece. Spectrochimica Acta - Part A, 56, 3-18.

Brook, G., Franco, N., Cherkinsky, A., Acevedo, A., Fiore, D., Pope, T., Weimar lii, R., Neher, G., Evans, H. y Salguero, T. (2018). Pigments, binders, and ages of rock art at Viuda Quenzana, Santa Cruz, Patagonia (Argentina). Journal of Archaeological Science: Reports, 21, 47-63.

Carden, N., Blanco, R., Poiré, D., Genazzini, C., Magnin, L. y García, P. (2014). Análisis de pigmentos del Macizo del Deseado: El abastecimiento de materias primas y la producción de pinturas rupestres en Cueva Maripe (Santa Cruz, Argentina). Relaciones de la Sociedad Argentina de Antropología, XXXIX(2), 483-508.

Cardich, A. (1979). A propósito de un motivo sobresaliente en las pinturas rupestres de "El Ceibo" (Provincia de Santa Cruz, Argentina). Relaciones de la Sociedad Argentina de Antropología, XIII, 163-182.

Chalmin, E., Castets, G., Delannoy, J., David, B., Barker, B., Lamb, L., Soufi, F., Pairis, S., Cersoy, S., Martinetto, P., Geneste, M., Hoerlé, S., Richards, T. y Gunn, R. (2017). Geochemical analysis of the painted panels at the "Genyornis" rock art site. Arnhem Land, Australia. Quaternary International, 430, 60-80.

Cueto, M., Frank, A. D. y Skarbun, F. (2018). Explotación de obsidianas en la Meseta Central de Santa Cruz. Estrategias de producción, uso y circulación. Chungara, 50(2), 235-253.

Domínguez, E. y Murray, H. (1997). The Lote 8 Kaolin Deposit, Santa Cruz, Argentina. Genesis and paper industrial application. En H. Kodama, A. M. Mermut y J. K. Torrance (Eds.), Proceedings of the 11th International Clay Conference. 57-64. Ottawa, Canadá.

Franchomme, J. M. (1991). L'art rupestre prehistorique de Patagonie [tesis doctoral inédita, Universidad de Bordeaux].

Frank, A. D. (2012). Tratamiento y daño térmico de artefactos líticos en los componentes tempranos del sitio Casa del Minero 1, Santa Cruz, Argentina. Chungara, 44(1), 25-37.

Gomes, H., Rosina, P., Holakooei, P., Solomon, T. y Vaccaro, C. (2013). Identification of pigments used in rock art paintings in GodeRoriso-Ethiopia using MicroRaman spectroscopy. Journal of Archaeological Science, 40, 4073-4082.
Gradin, C., Aschero, C. y Aguerre, A. M. (1979). Arqueologia del Área Río Pinturas (Santa Cruz). Relaciones de la Sociedad Argentina de Antropología, XIII, 183-228.

Iñíguez, A. y Gradin, C. (1977). Análisis mineralógico por difracciones de raxos $X$ de muestras de pinturas de la cueva de las manos, estancia Alto Río Pinturas (Provincia de Santa Cruz). Relaciones de la Sociedad Argentina de Antropología, XI, 121-128.

Marte, F., Careaga, V., Mastrángelo, N., De Faria, D. y Maier, M. (2014). The Sibyls from the church of San Pedro Telmo: A micro-Raman spectroscopic investigation. Journal of Raman Spectroscopy, 45, 1046-1051.

Panza, J. L. (1994). Descripción de la Hoja Geológica 4969-II. Tres Cerros Escala 1:250.000. Provincia de Santa Cruz. Boletin 213. Servicio Geológico Nacional.

Paunero, R. S., Frank, A. D., Skarbun, F., Rosales, G., Zapata, G., Cueto, M., Paunero, M., Martinez, D. G., López, R., Lunazzi, N. y Del Giorgio, M. (2005). Arte rupestre en estancia La María, Meseta Central de Santa Cruz: Sectorización y contextos arqueológicos. Relaciones de la Sociedad Argentina de Antropología, XXX, 147-168.

Prieto-Taboada, N., Gómez-Laserna, O., Martínez-Arkarazo, I., Olazabal, M. y Madariaga, J. (2014). Raman Spectra of the Different Phases in the CaSO4-H2O System. Analytical Chemistry, 86(20), 10.131-10.137.

Re, A. (2011). Representaciones rupestres en mesetas altas de la provincia de Santa Cruz. Circulación de información en espacios de uso estacional [tesis doctoral inédita, Universidad de Buenos Aires].

Sepúlveda, M. A. (2011). Pinturas rupestres y tecnología del color en el extremo sur de Chile. Magallania, 39(1), 193-210.

Skarbun, F. (2015). Estructura y explotación de los recursos líticos en el sector Meridional de la Meseta Central de Santa Cruz, Argentina. Magallania, 43, 191-210.

Tascon, M., Mastrángelo, N., Gheco, L., Gastaldi, M., Quesada, M. y Marte, F. (2016). Micro-spectroscopic analysis of pigments and carbonization layers on prehispanic rock art at the Oyola's caves, Argentina, using a stratigraphic approach. Microchemical Journal, 129, 297-304.

Wainwright, I., Helwig, K., Rolandi, D., Gradin, C., Podestá, M., Onetto, M. y Aschero, C. (2002). Rock paintings conservation and pigment analysis at Cueva de las Manos and Cerro de los Indios, Santa Cruz (Patagonia), Argentina. En ICCOM Comite for Conservation, 13th Triennial Meeting, Rio de Janeiro. Preprints, vol. II. (pp. 582-589). 
